\title{
Clinical, laboratory, and temporal predictors of neutralizing antibodies against SARS-CoV-2 among COVID-19 convalescent plasma donor candidates
}

\author{
Jim Boonyaratanakornkit, ${ }^{1,2,3}$ Chihiro Morishima, ${ }^{4}$ Stacy Selke, ${ }^{4}$ Danniel Zamora, ${ }^{1,2}$ Sarah McGuffin, ${ }^{1}$ Adrienne E. Shapiro, ${ }^{1,5}$ \\ Victoria L. Campbell, ${ }^{1}$ Christopher L. McClurkan, ${ }^{1}$ Lichen Jing, ${ }^{1}$ Robin Gross, ${ }^{6}$ Janie Liang, ${ }^{6}$ Elena Postnikova, ${ }^{6}$ Steven Mazur, ${ }^{6}$ \\ Vladimir V. Lukin, ${ }^{7}$ Anu Chaudhary, ${ }^{4}$ Marie K. Das, ${ }^{4}$ Susan L. Fink, ${ }^{4}$ Andrew Bryan, ${ }^{4}$ Alex L. Greninger, ${ }^{4}$ Keith R. Jerome, ${ }^{2,4}$ \\ Michael R. Holbrook, ${ }^{6}$ Terry B. Gernsheimer, ${ }^{1,3}$ Mark H. Wener, ${ }^{1,4}$ Anna Wald, ,2,8 and David M. Koelle ${ }^{1,2,4,5,9}$

\begin{abstract}
'Department of Medicine, University of Washington, Seattle, Washington, USA. Fred Hutchinson Cancer Research Center, Seattle, Washington, USA. ${ }^{3}$ Seattle Cancer Care Alliance, Seattle, Washington, USA. ${ }^{4}$ Department of Laboratory Medicine and Pathology, and ${ }^{5}$ Department of Global Health, University of Washington, Seattle, Washington, USA. ${ }^{6}$ Integrated Research Facility, Division of Clinical Research, National Institute of Allergy and Infectious Diseases (NIAID), NIH, Frederick, Maryland, USA. ' Kearney, Chicago, Illinois, USA. ${ }^{8}$ Department of Epidemiology, University of Washington, Seattle, Washington, USA. ${ }^{9}$ Benaroya Research Institute, Seattle, Washington, USA.
\end{abstract}

BACKGROUND. SARS-CoV-2-specific antibodies may protect from reinfection and disease, providing rationale for administration of plasma containing SARS-CoV-2-neutralizing antibodies (nAbs) as a treatment for COVID-19. Clinical factors and laboratory assays to streamline plasma donor selection, and the durability of nAb responses, are incompletely understood.

METHODS. Potential convalescent plasma donors with virologically documented SARS-CoV-2 infection were tested for serum IgC against SARS-CoV-2 spike protein S1 domain and against nucleoprotein (NP), and for nAb.

RESULTS. Among 250 consecutive persons, including 27 (11\%) requiring hospitalization, who were studied a median of 67 days since symptom onset, $97 \%$ were seropositive on 1 or more assays. Sixty percent of donors had $n A b$ titers $\geq 1: 80$. Correlates of higher nAb titers included older age (adjusted OR [AOR] 1.03 per year of age, $95 \% \mathrm{Cl} 1.00-1.06$ ), male sex (AOR 2.08, 95\% Cl 1.13-3.82), fever during illness (AOR 2.73, 95\% Cl 1.25-5.97), and disease severity represented by hospitalization (AOR 6.59, 95\% Cl 1.32-32.96). Receiver operating characteristic analyses of anti-S1 and anti-NP antibody results yielded cutoffs that corresponded well with nAb titers, with the anti-S1 assay being slightly more predictive. nAb titers declined in 37 of 41 paired specimens collected a median of 98 days (range $77-120)$ apart $(P<0.001)$. Seven individuals $(2.8 \%)$ were persistently seronegative and lacked T cell responses.

CONCLUSION. nAb titers correlated with COVID-19 severity, age, and sex. SARS-CoV-2 IgC results can serve as useful surrogates for nAb testing. Functional nAb levels declined, and a small proportion of convalescent individuals lacked adaptive immune responses.

FUNDING. The project was supported by the Frederick National Laboratory for Cancer Research with support from the NIAID under contract number 75N91019D00024, and was supported by the Fred Hutchinson Joel Meyers Endowment, FastGrants, a New Investigator award from the American Society for Transplantation and Cellular Therapy, and NIH contracts 75N93019C0063, 75N91019D00024, and HHSN272201800013C, and NIH grants T32-AI118690, T32-AI007044, K08-AI119142, and K23-Al140918.

Authorship note: JB and CM and $\mathrm{AW}$ and DMK contributed equally to this work. Conflict of interest: ALG receives consulting fees from Abbott Molecular. RG, JL, and MRH performed this work as employees of Laulima Government Solutions LLC. EP, an employee of Tunnell Government Services Inc., is a subcontractor to Laulima Government Solutions LLC.

Copyright: $(2021$, American Society for Clinical Investigation.

Submitted: October 7, 2020; Accepted: December 9, 2020 ;

Published: February 1, 2021

Reference information: J Clin Invest. 2021;131(3):e144930.

https://doi.org/10.1172/JCl144930.

\section{Introduction}

Since its emergence in late 2019, severe acute respiratory syndrome coronavirus 2 (SARS-CoV-2) infection has spread globally, with devastating effects in many communities. At this time, remdesivir remains the only therapeutic approved by the Food and Drug Administration (FDA) for the treatment of SARS-CoV-2 infection. No vaccines for the prevention of SARS-CoV-2 have been fully approved by the FDA yet. Passive immunotherapy with convalescent plasma, hyperimmune $\gamma$-globulin, or monoclonal 


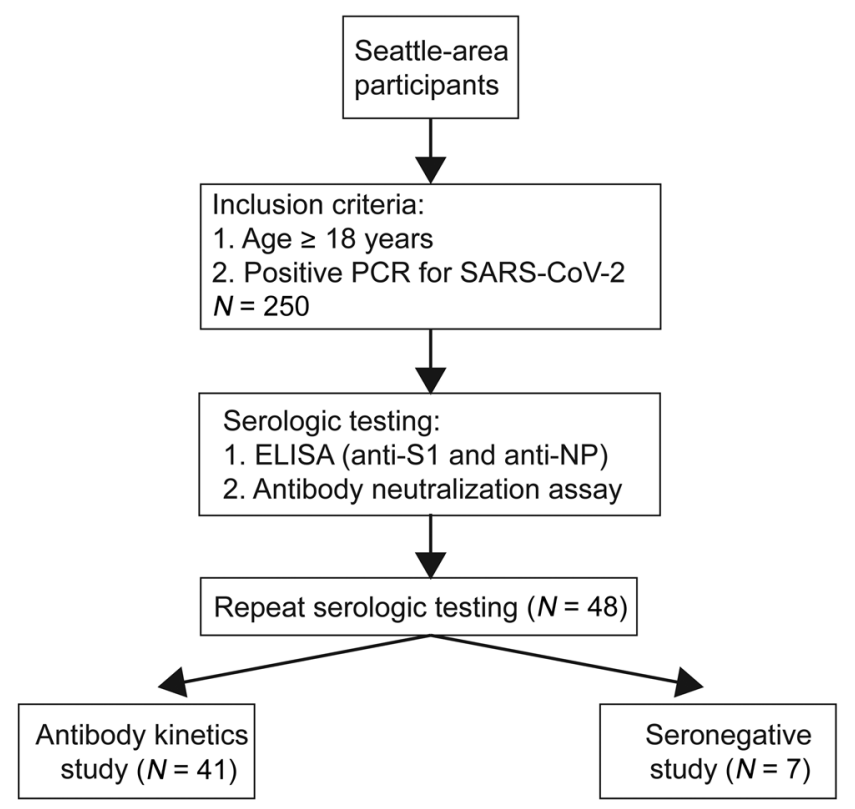

Figure 1. Study design and participants.

antibodies is beneficial for treatment or prophylaxis of several infections (1), and these approaches are under investigation in patients with COVID-19. Both single-donor and pooled immunoglobulin products currently prioritize collection of convalescent donor plasma with high levels of neutralizing antibodies (nAbs).

$\mathrm{nAb}$ assays are challenging to perform and have biosafety concerns. A better understanding of the predictors and correlates of high $\mathrm{nAb}$ titers could be useful for improving access to highquality therapeutic plasma products. In addition, it is uncertain how durable acquired immunity will be in response to SARSCoV-2 infection. Protection from several other respiratory viruses after natural infection is temporary and incomplete $(2,3)$, and longitudinal information concerning nAb levels may assist modeling of the future of the outbreak until effective vaccines become widely used. We evaluated antibody levels in candidate plasma donors recovered from virologically documented SARS-CoV-2 infection and examined the trajectory of antibody levels in a subset of donors over time. Further, we compared nAb titers with 2 rapid commercially available assays to determine whether these assays could substitute for time- and labor-intensive nAb measurements that require a biosafety level 3 facility.

\section{Results}

Participant characteristics. We enrolled 250 consecutive persons interested in convalescent plasma donation (Figure 1) with a median age of 51 years (range 19-91); 48\% were men (Table 1). The median duration of COVID-19 symptoms was 15 days (range 0-49), and the median number of days between positive PCR testing and initial antibody testing was 61 days (range 14-112). Twenty-seven participants (10.8\%) reported hospitalization, including 9 (3.6\%) who received ICU-level care. The most common symptoms reported were fatigue (86\%), fever (74\%), muscle aches $(73 \%)$, cough (72\%), anosmia/ageusia (61\%), and headache (60\%) (Supplemental Figure 1A; supplemental material available online with this arti- cle; https://doi.org/10.1172/JCI144930DS1). The most commonly reported underlying comorbidities were lung disease (14.4\%), heart disease (13.6\%), hypertension (12.8\%), cancer (12.8\%), hyperlipidemia (9.6\%), and diabetes (9.6\%) (Supplemental Figure 1B).

Clinical correlates of $n A b$ response. Among the 250 participants, $12.8 \%$ had nAb titers below 1:40, 27.6\% had titers of 1:40, 34.4\% had titers of $1: 80$, and $25.2 \%$ had titers of $1: 160$ or greater (Figure 2). Next, we examined the clinical correlates of nAb titers of 1:80 or greater, as this group was eligible for donation to a pooled immunoglobulin product protocol. In a univariable analysis, older age (OR 1.04 per year, 95\% CI 1.02-1.06), male sex (OR 2.27, 95\% CI 1.35-3.82), the presence of fever (OR 3.12, 95\% CI 1.73-5.61) or difficulty breathing (OR 2.12, 95\% CI 1.26-3.54) during the acute illness episode, hospitalization denoting more severe disease (OR 9.98, 95\% CI 2.31-43.16), and having diabetes (OR 5.36, 95\% CI 1.55-18.48) were significantly associated with higher nAb titers (Table 2). In a multivariable analysis, older age (adjusted OR [AOR] 1.03, 95\% CI 1.00-1.06), male sex (AOR 2.08, 95\% CI 1.13-3.82), the presence of fever during the acute illness episode (AOR 2.73, 95\% CI 1.25-5.97), and hospitalization (AOR 6.59, 95\% CI 1.32-32.96) remained significantly associated with higher $\mathrm{nAb}$ titers (Table 2). A longer interval in time between the diagnostic PCR and antibody testing was also associated with lower nAb titers (OR 0.97, 95\% CI 0.96-0.99) (Table 2).

Comparison of anti-SARS-CoV-2 antibody measurements. Circulating IgG antibodies against SARS-CoV-2 antigens were measured using IgG immunoassays for anti-S1 (Euroimmun) and anti-nucleoprotein (anti-NP) (Abbott). Among the 250 sera analyzed at the initial time point, 23 samples (9.2\%) were negative by the manufacturer's criteria versus $11(4.4 \%)$ by a $z$ score threshold

\section{Table 1. Demographic and clinical characteristics of study subjects}

\begin{tabular}{|c|c|}
\hline Category & Value $(n=250)$ \\
\hline Median age in years (range) & $51(19-91)$ \\
\hline Men & $119(47.6 \%)$ \\
\hline Women & $131(52.4 \%)$ \\
\hline Hispanic ethnicity & $11(4.4 \%)$ \\
\hline \multicolumn{2}{|l|}{ Race } \\
\hline White & $214(85.6 \%)$ \\
\hline Asian & $22(8.8 \%)$ \\
\hline Black & $4(1.6 \%)$ \\
\hline Pacific Islander & $1(0.4 \%)$ \\
\hline Native American & $1(0.4 \%)$ \\
\hline Other ${ }^{A}$ & $4(1.6 \%)$ \\
\hline More than 1 race & $4(1.6 \%)$ \\
\hline Median (range) days between symptom resolution and antibody test ${ }^{\mathrm{B}}$ & $50(21-110)$ \\
\hline Median (range) days between positive PCR and antibody test & $61(14-112)$ \\
\hline \multicolumn{2}{|l|}{ Severity } \\
\hline Outpatient $^{\mathrm{C}}$ & $223(89.2 \%)$ \\
\hline Hospitalized but not in ICU & $18(7.2 \%)$ \\
\hline ICU & $9(3.6 \%)$ \\
\hline \multicolumn{2}{|c|}{$\begin{array}{l}\text { A“Other" categorization was made by the participant. }{ }^{\text {BExcluding } 1} \\
\text { asymptomatic person. Includes } 1 \text { asymptomatic participant who was a } \\
\text { household contact of a person with symptomatic COVID-19. }\end{array}$} \\
\hline
\end{tabular}




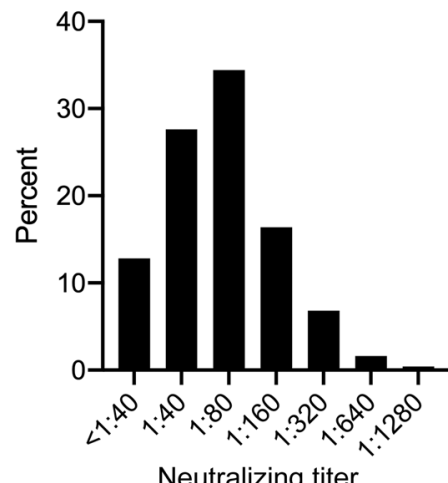

Figure 2. Distribution of nAb titers in convalescent subjects $(n=250)$. Values on the $x$ axis represent the dilution factor of serum that yielded a $50 \%$ reduction in SARS-CoV-2 infection of Vero E6 cells.

of 3.0 for the Abbott assay. For the Euroimmun assay, 20 (8.0\%) were negative by a $z$ score threshold of 3.0 versus $30(12.0 \%)$ by the manufacturer's criteria. A minority of samples demonstrated discordant results (Figure 3); overall, 15 (6\%) were discordant for seropositivity using $z$ score thresholds of 3.0 versus 19 (7.6\%) using manufacturers' thresholds.
Quantitative assay results were generally concordant. $\mathrm{nAb}$ titers correlated with $z$ scores from the anti-S1 Euroimmun assay (Pearson's correlation 0.49, $P<0.001$ ) and from the Abbott anti$\mathrm{NP}$ assay (Pearson correlation 0.39, $P<0.001$ ) (Figure 3 , A and B). $Z$ scores of the Euroimmun and Abbott assays also correlated with each other (Pearson's correlation 0.69, $P<0.001$ ) (Figure 3C). Receiver operating characteristic curves were generated to summarize the sensitivity and specificity of the Abbott and the Euroimmun assays for detecting $\mathrm{nAb}$ titers $\geq 1: 40, \geq 1: 80$, and $\geq 1: 160$ (Supplemental Figure 2). The area under the curve (AUC) for the Euroimmun assay was slightly greater than that for the Abbott assay across all $3 \mathrm{nAb}$ levels.

The criteria for positivity in the binding IgG tests were subjected to further analysis focusing on nAb cutoffs of 1:80 or higher. $\mathrm{A} z$ score of 3.0 in the Euroimmun assay had a sensitivity of $99 \%$ and a specificity of $17 \%$ for an $n A b$ titer $\geq 1: 80$ (Table 3 and Supplemental Figure 2B). To estimate optimal cutoffs, we used Youden's index (4), which indicated that at an optimized $z$ score cutoff of 32.6, the Euroimmun assay had a sensitivity of $77 \%$ and a specificity of $81 \%$. A $z$ score of 3.0 in the Abbott assay had a sensitivity of $100 \%$ but a specificity of only $11 \%$. Using an optimized $z$ score cutoff of 34.2 , the Abbott assay had a sensitivity of $78 \%$ and a specificity of $72 \%$. Because the optimal therapeutic titer of convalescent plasma

\section{Table 2. Clinical correlates of high ( $\geq 1: 80$ ) circulating SARS-CoV-2 nAb titers}

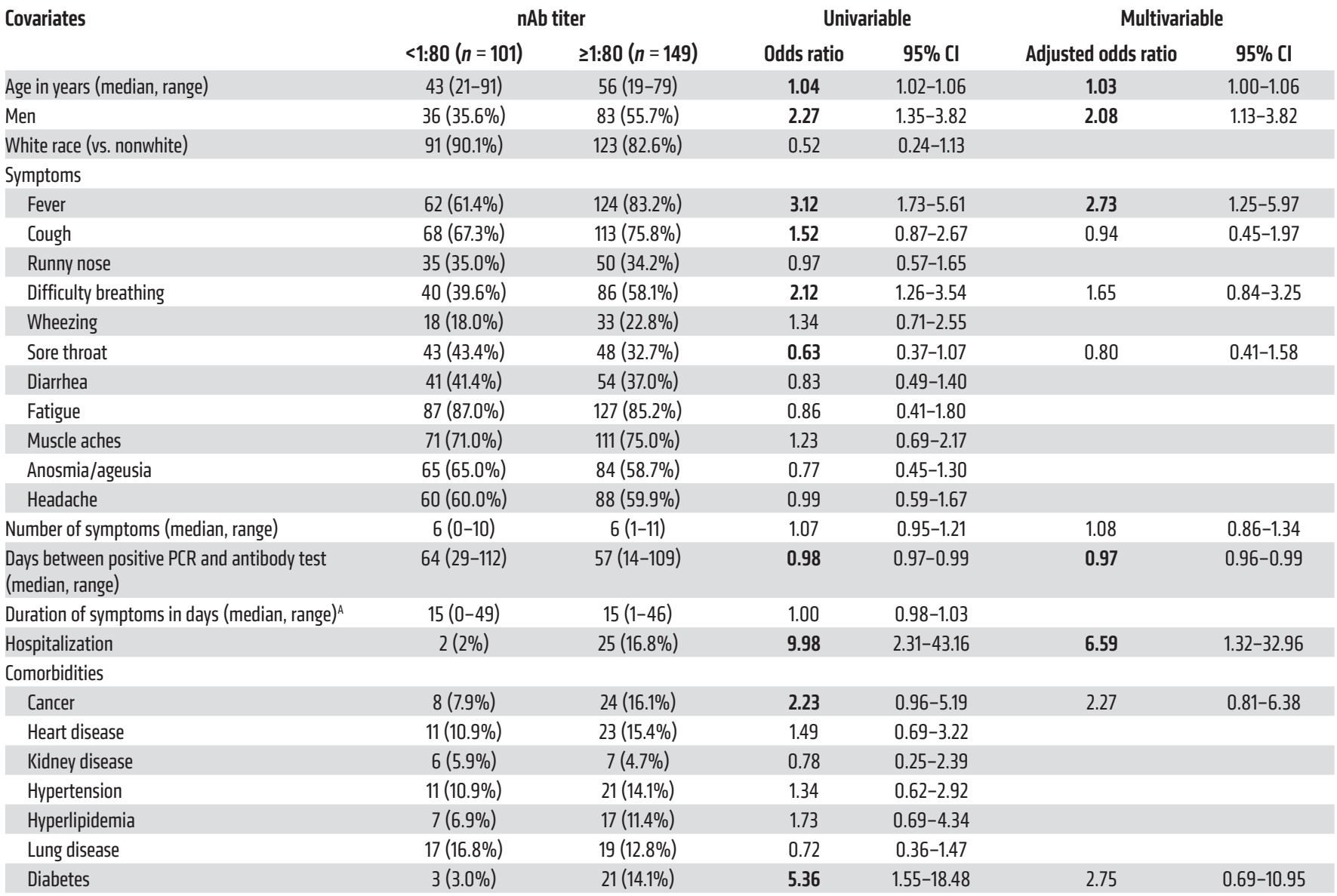

Odds ratios in boldface indicate $P<0.2$ in the univariable analysis or $P<0.05$ in the multivariable analysis. ${ }^{A}$ Excluding 1 asymptomatic subject. 

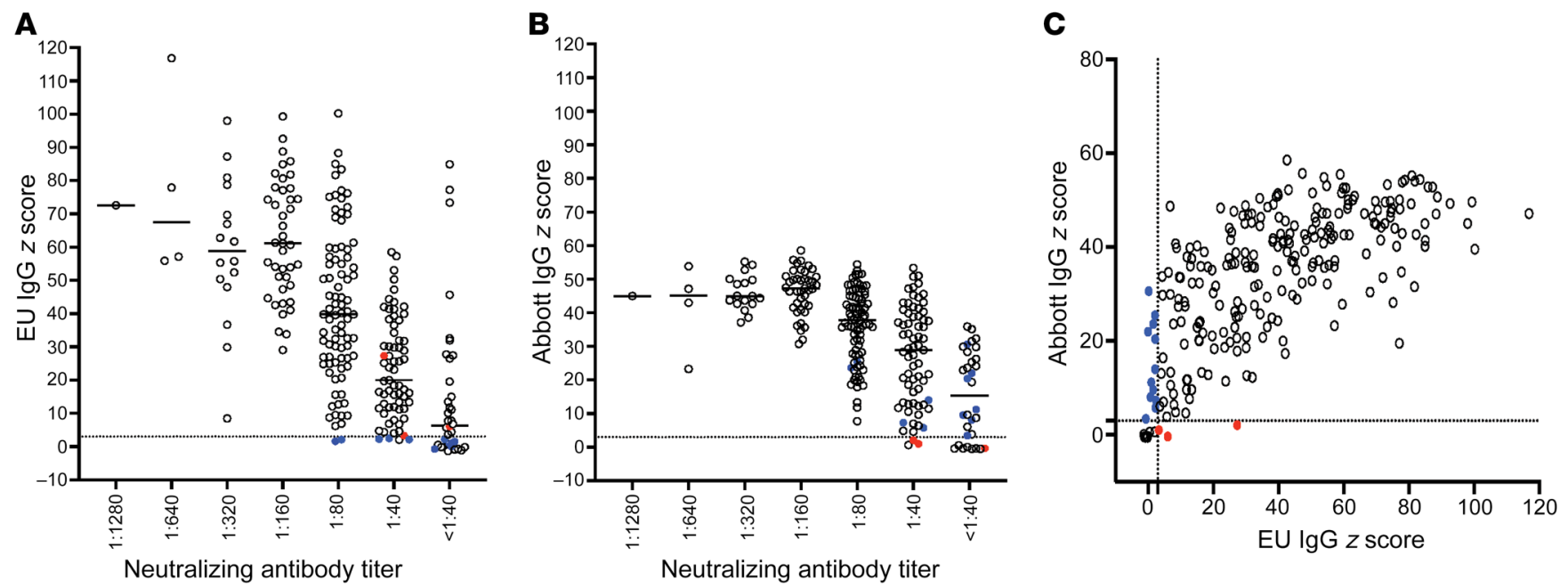

Figure 3. Relationship between serum measurements of anti-SARS-CoV-2 antibodies. Levels of anti-SARS-CoV-2 IgG antibodies specific for S1 (Euroimmun; EU) (A) or of anti-SARS-CoV-2 IgG antibodies specific for NP (Abbott) expressed as z scores (B) are shown relative to nAb titers obtained from the same samples. Each symbol represents the initial specimen from one person. (C) Anti-SARS-CoV-2 S1 IgG levels (EU) relative to anti-SARS-CoV-2 nucleocapsid protein (NP) IgG levels (Abbott). The dotted lines indicate the positivity threshold ( $z$ score of 3 ) for both EU and Abbott assay results. Colored circles indicate discordant results between the Euroimmun and Abbott assays. Blue circles represent samples with negative $z$ score results (<3.0) by Euroimmun and positive $z$ score results ( $\geq 3.0$ ) by Abbott. Conversely, red circles represent samples with positive results $(z \geq 3.0)$ by Euroimmun and negative results $(z<3.0)$ by Abbott.

is unknown, we also calculated the sensitivity and specificity of both IgG binding assays to predict nAb titers $\geq 1: 160$; as expected, these $z$ scores were higher (Table 3).

Antibody titers over time after recovery from COVID-19. Lower nAb titers were associated with a longer time after symptom resolution according to our multivariable analysis (Table 2). In a cross-sectional visualization of serologic data from persons with an nAb titer of 1:40 or greater at the initial blood draw, nAb titers, anti-S1 IgG levels, and anti-NP IgG levels all demonstrated trends toward lower antibody levels with increased interval between symptoms and blood sampling (Supplemental Figure 3). To further investigate this initial observation, 41 participants enrolled during April 2020 who had nAb titers of 1:40 or greater returned for a second blood draw to examine antibody kinetics. Neutralization titers decreased over time in all but 4 participants. From these data, nAb half-life was calculated at 66.2 days (95\% CI 55.5-82.5)
(Figure 4A). A decline in anti-S1 IgG and anti-NP IgG levels over time was also observed in $83 \%$ and $98 \%$ of participants, respectively (Figures 4, B and C). Among the 7 hospitalized participants who had repeat testing, all had lower antibody titers at the second time point on neutralization, anti-S1, and anti-NP assays. Given our observation that compression of optical density ratios and resultant $z$ scores occurs at higher levels of IgG, half-life calculations were not performed for these latter assays.

Clinical characteristics and repeat serologic results from seronegative participants. We identified 7 participants who lacked circulating antibody responses by all 3 assays, despite having documented SARS-CoV-2 infection. Among these, 1 had been asymptomatic and 1 had been hospitalized for COVID-19 (Table 4). Five (71\%) were women with a median age of 48 (range 40-62). The median time between positive SARS-CoV-2 PCR and antibody testing was 42 days (range $30-87$ ). The previously hos-

Table 3. Sensitivity and specificity of circulating IgC anti-S1 and anti-NP immunoassays for predicting nAb titers

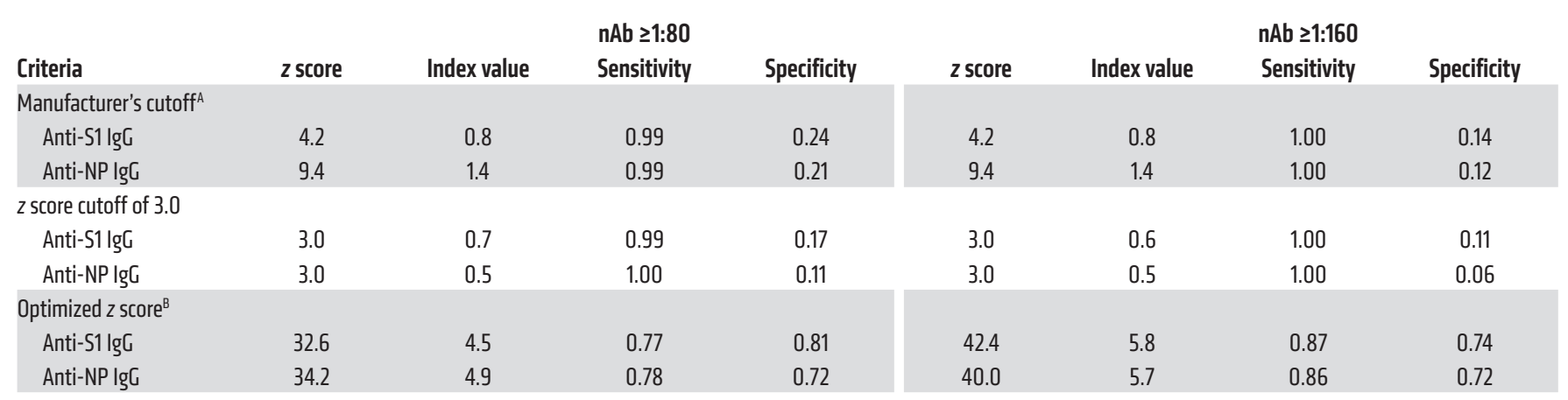

${ }^{A} z$ scores calculated for manufacturer-determined $\mathrm{OD}$ ratio positivity thresholds of 0.8 for anti-S1 IgG and 1.4 for anti-NP IgG immunoassays. ${ }^{\mathrm{B}} \mathrm{Optimal} z$ score based on the maximum value of Youden's index. 
A

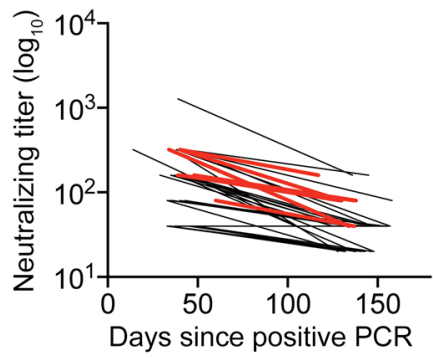

B

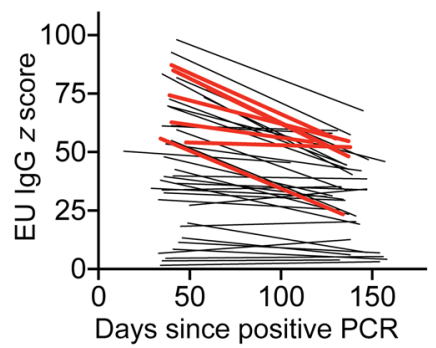

C

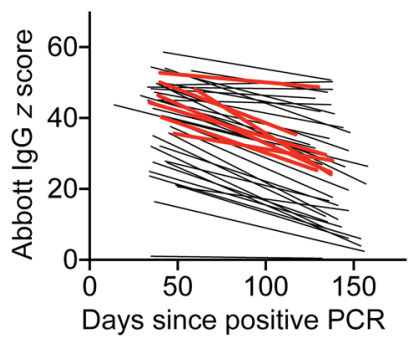

Figure 4. Individual antibody levels obtained at various time points after symptom resolution from 41 persons recovered from PCR-positive SARSCoV-2 infection and with an initial nAb titer of 1:40 or greater. The same 41 persons are represented in each graph. Red lines correspond to hospitalized participants $(n=7)$. Neutralization assay (A), Euroimmun (anti-S1) IgG assay (B), and Abbott (anti-NP) IgG assay (C) results as a function of time since resolution of symptoms. Each line connects 2 results from a single participant.

pitalized participant with consistently negative antibody results across all 3 antibody tests had received infliximab for vasculitis 1 week before becoming infected with H1N1 influenza, followed 1 week later by infection with SARS-CoV-2. Six seronegative participants had a repeat blood draw, a median time of 43 days (range 20-55) after the first. Serologic test results remained negative in all individuals by all 3 assays (Table 4 ).

To further investigate the immune status of the seronegative individuals, we performed IFN- $\gamma$ ELISPOT using PBMCs from follow-up samples of the 6 persons with repeat blood draws, and peptides from SARS-CoV-2 structural proteins. Previous work has shown that the majority of $\mathrm{T}$ cell responses in recovered COVID-19 patients recognize these viral proteins (5). As comparators, we tested a subset of seropositive persons and pre-2019 healthy donors. We detected robust $\mathrm{T}$ cell responses in PBMCs from nearly all convalescent SARS-CoV-2-seropositive individuals, whereas $\mathrm{T}$ cell responses from SARS-CoV-2-seronegative patients were similar to those from healthy donors and were below a provisional cutoff of 100 IFN- $\gamma$ spot-forming units per million PBMCs. Only 1 of 16 seropositive COVID-19 participants had $\mathrm{T}$ cell responses below this level (Supplemental Figure 4).

\section{Discussion}

Our characterization of a large cohort of potential SARS-CoV-2 convalescent plasma donors provides several useful observations relevant to protection after natural infection or vaccination and the manufacture of antibody-based therapeutics. We found that a significant proportion (40\%) of persons failed to meet a protocoldriven $\mathrm{nAb}$ threshold ( $\geq 1: 80$ ) for contribution to a pooled immunoglobulin product. Clinical factors associated with higher levels of nAb, specifically male sex, older age, higher disease severity, and shorter interval from recovery, were identified; this information could streamline future donor recruitment. Repeat testing demonstrated a decline of functional nAb over time, suggesting that these clinical characteristics are most valuable when used early after recovery. We also found that anti-SARS-CoV-2 IgG results from 2 widely accessible and commercially available immunoassays could reliably predict high nAb titers when used relatively early after COVID-19. Test cutoffs were empirically derived that can assist in selecting donors with increased likelihood of having high nAb titers. Finally, approximately $3 \%$ of our cohort, all with documented SARS-CoV-2 infection, did not exhibit adaptive immune responses in multiple antibody and $\mathrm{T}$ cell assays.

Pending full approval of antiviral drugs or a preventative vaccine, much effort has focused on passive immunization for the treatment or prevention of COVID-19, ranging from singledonor plasma and pooled immunoglobulin products to recombinant antibodies (6-8). Pooled products have a track record of safety and efficacy for other pathogens, including respiratory viruses (1), and are most efficiently manufactured from plasma containing higher amounts of functional antibody. While the immunoglobulin isotypes and functional activities associated with

Table 4. Clinical and laboratory characteristics of seronegative individuals

\begin{tabular}{|c|c|c|c|c|c|c|c|c|}
\hline Subject & $\begin{array}{c}\text { Age at } \\
\text { blood draw }\end{array}$ & Sex & Severity & Comorbidities & $\begin{array}{l}\text { Days sx onset } \\
\text { to 1st draw }\end{array}$ & $\begin{array}{l}\text { Days (+) PCR } \\
\text { to 1st draw }\end{array}$ & $\begin{array}{c}\text { Days sx resolved } \\
\text { to 1st draw }\end{array}$ & $\begin{array}{l}\text { Days between 1st } \\
\text { and } 2 \text { nd draws }\end{array}$ \\
\hline 2 & 48 & M & Outpt & None & 43 & 39 & 28 & 55 \\
\hline 4 & 48 & $\mathrm{~F}$ & Outpt & None & 42 & 42 & 22 & 41 \\
\hline 5 & $40^{A}$ & $\mathrm{~F}$ & Outpt (Asx) & Asthma & 52 & 50 & 40 & NA \\
\hline 6 & 45 & $\mathrm{~F}$ & Inpt & Lung carcinoid; asthma; vasculitis & 95 & 87 & 70 & 20 \\
\hline
\end{tabular}

ASubject did not return for a second blood draw. Asx, asymptomatic; Inpt, inpatient; Outpt, outpatient; sx, symptoms. 
improved COVID-19 outcomes remain an active area of investigation (9), neutralization of viral entry is thought to be a desirable attribute that corresponds to protection. Thus, qualifying plasma donation for NIH hyperimmune globulin protocol NCT0 4344977 has focused on high nAb titers. Observational data from a singledonor plasma program generally support the premise that nAbs are desirable, with lower mortality rates noted for persons receiving plasma containing the top quintile of anti-S binding antibodies (6). A small randomized controlled trial also found a trend toward a shorter time to clinical improvement and lower mortality with convalescent plasma (10). Several studies of neutralizing monoclonal antibodies in animal models and a study of an outbreak on a ship further support the importance of nAbs in protection (11-14). We therefore sought to identify clinical characteristics associated with plasma nAbs and compared 2 widely used SARS-CoV-2 antibody tests for ability to predict nAb titers of 1:80 or greater.

Our clinical findings of an association of higher nAb titers with male sex, older age, and hospitalization are consistent with a recent report from another convalescent plasma donor candidate study (15) and other reports concerning recovered individuals (16-18). While the study by Klein et al. (15) used similar assays (nAb and anti-spike) and found similar associations with higher nAb titers, our study included a larger sample size ( $n=250$ vs. 126), as well as results from a second widely available clinical serologic assay to detect anti-NP antibodies. We also performed a sensitivity analysis comparing anti-NP and anti-S1 titers for predicting nAb titers, longitudinally analyzed $\mathrm{nAb}$ titers, and examined $\mathrm{T}$ cell responses in apparently seronegative individuals. The relationship of greater infection severity, and presumably greater antigen load, with higher acquired B cell responses was observed in both convalescent plasma donor studies. This observation is paralleled by data from others showing an association between more severe infection and higher $\mathrm{T}$ cell responses in general, and, in particular, circulating $\mathrm{CD} 4^{+} \mathrm{T}$ follicular helper (Tfh) responses (19). Tfh cells provide critical positive signals to antigen-specific B cells in lymph node germinal center reactions, promoting avid and long-lasting antibody responses.

Our cross-sectional analyses also found a trend toward lower nAb titers with increased elapsed time between symptom resolution and antibody testing. This was confirmed in longitudinal analyses of 41 participants reevaluated approximately 3 months after their first test. A quarter of the reevaluated participants had nAb titers below 1:40 at the second time point, and declines in circulating anti-S1 and anti-NP IgG levels were found in more than $80 \%$ of participants over the relatively short period of time between the 2 blood draws. A critical and currently unresolved issue for SARS-CoV-2 infection is the extent to which prior infection prevents or ameliorates various characteristics of reinfection, including disease, viral shedding, and transmission potential. It is likely that protection efficiency will vary according to clinical characteristics of the host and of the initial infection, with time after initial infection, and with the virologic or clinical endpoint under consideration. The issue of antibody durability has been addressed in prior cross-sectional and longitudinal studies, obviously limited by the recency of the pandemic. Decreases in SARSCoV-2 nAb titers have been observed in some prior studies (17, 20-22) but not others (23). The decline of nAb titers over time and the low somatic hypermutation in immunoglobulin genes recovered from SARS-CoV-2-specific memory B cells (24) both suggest that ineffective help from $\mathrm{Tfh} \mathrm{CD}^{+} \mathrm{T}$ cells is occurring in germinal centers. While Tfh-like SARS-CoV-2-reactive $\mathrm{CD} 4^{+} \mathrm{T}$ cells have been detected in the blood (19), autopsy data from fatal COVID-19 are consistent with poor germinal center reactions (25). Overall, waning immunity after natural infection raises concern for reinfection over time as observed with other respiratory viruses, including the endemic coronaviruses $(2,3)$. Data on protection from reinfection with SARS-CoV-2 are incomplete at this time, with case reports of reinfection (26) contrasting with cohort data from an occupational outbreak in which persons with baseline SARS-CoV-2-specific circulating antibody were protected from infection (11). While this study focused on $\mathrm{nAb}$, we recognize that other investigators have identified other functional antibody characteristics that may correlate with infection severity (9), and it is not known how these features correlate with protection. Additional research will be required to address the immunologic and clinical parameters associated with protection from reinfection after SARS-CoV-2 infection.

With regard to test performance, we found a slightly stronger correlation between nAb titers and anti-S1 IgG levels using the Euroimmun assay compared with anti-NP IgG levels determined using the Abbott assay. We were able to establish cutoffs using $z$ scores for each assay that had good performance for predicting nAb titers of 1:80 or greater, or 1:160 or greater, which should be useful as the clinical efficacy of single-donor and pooled immunoglobulin products continues to be defined. The superior performance of the anti-S1 assay is not unexpected given that antibodies that neutralize SARS-CoV-2 bind to the S protein present on the surface of infectious virus. This finding is also consistent with studies that have shown that the detection of NP-binding antibodies does not always correlate with the presence of $\mathrm{nAb}$ (27). Although we did not examine antibodies against the M or E proteins of SARS-CoV-2, these antibodies are thought to play little if any role in viral neutralization (28).

In the present study, we used $z$ scores to standardize serologic results to the same scale and to account for local test performance characteristics (29). Despite using a relatively conservative positivity threshold of 3 SDs above our assay-specific negative control means, these $z$ score positivity thresholds were lower than manufacturer-recommended thresholds for both assays. Accordingly, a larger number of samples were considered "negative" by both assays using manufacturer-recommended thresholds compared with $z$ scores. Although continuous values are obtained and accessible for both Abbott and EU assays, it is worth noting that both are Emergency Use Authorized as qualitative and not quantitative assays. Although the Euroimmun assay appears to have a wider analytic range in comparison with the Abbott assay, both assays yield results that plateau at different thresholds. Thus, values at higher levels for both assays would require dilution of high-titer sera to achieve linear results. Even in the absence of reassay after dilution, however, we were able to discern declines in binding IgG titers over time within subjects, similar to our observations for $\mathrm{nAb}$.

A small proportion (3\%) of participants tested negative on all 3 antibody assays despite SARS-CoV-2 RNA detection in previously collected respiratory specimens. Each seronegative COVID-19recovered patient tested also had low $\mathrm{T}$ cell responses, similar to 
pre-2019 healthy controls, while most seropositive subjects had abundant IFN- $\gamma$-producing cells in the blood, similar to other reports (30). While false-positive PCR results are possible, all subjects except one, who was a household contact of a person with symptomatic and PCR-confirmed COVID-19, had a clinical syndrome consistent with COVID-19. Asymptomatic and mildly symptomatic patients with COVID-19 have been found to have lower antibody levels and also were more likely to serorevert in the early convalescent phase (18). Discordance between positive PCR and lack of circulating antibody may be related to timing of the blood draw after illness, milder symptoms correlating with lower or briefer antigen exposure, and/or an unrecognized immune suppression.

We consider our T cell IFN- $\gamma$ results to be preliminary, as we studied a small number of individuals in comparison with our antibody assays. We set a provisional cutoff near 100 IFN- $\gamma$ spot-forming units per million PBMCs, with all seronegative COVID-19 participants and healthy donors below this level. One seropositive participant had a low ELISPOT result in this range, which could be explained by several possibilities. Our ELISPOT assay included only a portion of the SARS-CoV-2 proteome (nucleocapsid, spike, and membrane). Others have shown $\mathrm{T}$ cell reactivity to SARS-CoV-2 proteins not present in our assay (31). The timing of blood collection could also have been a factor, as the low ELISPOT result was obtained from blood drawn 6 weeks after the participant's positive serology test. Serologic results also reverted to negative in the later blood sample obtained for ELISPOT. Finally, it is possible that this participant had a true dissociation between antibody and $\mathrm{T}$ cell responses, as previously reported (32). Additional studies with larger cohorts will be necessary to establish firm cutoffs for ELISPOT results; such results may also vary with the interval between recovery and specimen collection in the absence of re-exposure or vaccination.

Our study has some limitations. First, although our sample population included a clinically diverse population with respect to age, sex, and clinical presentation, the cohort was predominantly white. Additional studies in minority populations at risk for worse outcomes will be needed to determine the generalizability of these results. As would be expected for a convalescent plasma study given the disease course of COVID-19, most plasma donor candidates did not require hospitalization during their acute illness. Second, our study was mainly cross-sectional, and longitudinal data were obtained on only a subset of the cohort. Third, in analyzing the decline of nAb titers, a titer of 1:20 was used as a surrogate for all titers less than 1:40, since this value was the next serial 2 -fold titer below the lowest positive result. We chose this conservative surrogate value to avoid the possibility of overestimating the rate of decline in nAb titers. Fourth, we focused on blood immune responses. It is possible that the seronegative individuals in our study mounted a mucosal humoral or $\mathrm{T}$ cell response that could provide protection against reinfection. Probing mucosal and $\mathrm{T}$ cell immunity may be important in understanding the immune response of subjects with milder infection.

In summary, our data provide important information regarding the predictive value of clinical factors and commercially available immunoassay results for high $\mathrm{nAb}$ titers. This information could aid in streamlining the selection of potential convalescent plasma donors and increase access to and efficiency of donor sample testing. Since transfusion of plasma with higher antibody titers has been associated with improved outcomes (6), and since higher-titer plasma is the most suitable for the creation of pooled products, our data further suggest that plasma donations should be sought closer to the resolution of symptoms, rather than later, to optimize yield. A minimum level of $\mathrm{nAb}$ necessary for protection has not been established, and it is unknown whether persons with low titers will boost their immune responses upon re-exposure. However, our data raise concern that functional antibodies show a quantitative decline over a relatively short period of time after recovery in those with relatively mild infections. Further insight into these issues is critical to monitoring of herd immunity and implementation of immunization programs.

\section{Methods}

Study description. Seattle-area participants were recruited for potential donation of single-donor plasma units (ClinicalTrials.gov: NCT04338360), and plasma for manufacture of a pooled anti-SARSCoV-2 product (NCT04344977). Both studies required age 18 or greater and PCR positivity to SARS-CoV-2; criteria for donation for the pooled product also included an nAb titer of $\geq 1: 80$ (33). Participants provided demographic and clinical information, including positive SARS-CoV-2 results, and a blood sample.

Serologic assays. Plasma from EDTA and heparinized blood and serum were prepared within 12 hours of collection. Serum and plasma for IgG assays were stored at $4^{\circ} \mathrm{C}$ and assays performed within 72 hours of sample processing. PBMCs were isolated from heparinized blood within 12 hours of phlebotomy using standard Ficoll-Hypaque density gradient centrifugation and cryopreserved in liquid nitrogen in 90\% FCS $/ 10 \%$ DMSO. EDTA plasma was stored at $-80^{\circ} \mathrm{C}$ for neutralization assays.

Serologic testing was performed using 2 FDA-authorized (via Emergency Use Authorization), CE-marked immunoassays; both are approved to provide qualitative results, although both yield quantitative values. The anti-SARS-CoV-2 IgG ELISA from Euroimmun (Lubeck, Germany) measures antibodies against recombinant spike protein (S1 domain), containing the receptor binding domain that interacts with angiotensin converting enzyme 2 (ACE2) (34). All testing and analyses were performed according to the manufacturer's protocols, with the optical density ratio (ODR) calculated using the kit calibrator. The manufacturer-provided reference range is as follows: ratio $<0.8$, negative; ratio 0.8 to $<1.1$, borderline; and ratio $\geq 1.1$, positive. The Abbott SARS-CoV-2 IgG chemiluminescent microparticle immunoassay (Abbott ARCHITECT) measuring antibodies against the SARS-CoV-2 nucleoprotein (NP) was performed per the manufacturer's instructions. Equivalent to an ODR, the kit calibrator was used to establish the assay index result. Index values $\geq 1.4$ are considered positive by the manufacturer.

To standardize results and facilitate comparisons, ODR scores for each sample were converted to $z$ scores (number of SDs above the negative control mean) as follows: $z$ score $=$ (test ODR - mean negative control ODR) / mean negative control SD (29). Negative control sera had been collected between 2015 and 2019 from healthy community blood donors and from patients tested in the clinical laboratory by Western blot for potential HSV infection $(n=78$ for Euroimmun and $n=136$ for Abbott). ODRs were converted to $z$ scores as follows: for Euroimmun, $z$ score $=(\mathrm{ODR}-0.26) / 0.13$; and for Abbott, $z$ score $=$ (index result -0.08)/0.14. A conservative $z$ score $\geq 3$ was considered positive to minimize false-positive results. 
$\mathrm{nAb}$ titers were performed using a fluorescence reduction neutralization assay with authentic SARS-CoV-2 (2019-nCoV/USA-WA1A12/2020 from the US Centers for Disease Control and Prevention, Atlanta, Georgia, USA) at the NIH/National Institute of Allergy and Infectious Diseases (NIAID) Integrated Research Facility at Fort Detrick, Maryland, USA. In these experiments, a fixed volume of diluted virus was incubated with an equivalent volume of test plasma for 1 hour at $37^{\circ} \mathrm{C}$ before addition of the suspension to Vero E6 cells (ATCC, CRL-1586). The virus was allowed to propagate for 24 hours prior to fixation of the cells. Following fixation, the cells were permeabilized and probed with a SARS-CoV-2 nucleoprotein-specific rabbit primary antibody (Sino Biological, 40143-R001) followed by an Alexa Fluor 594conjugated secondary antibody (Life Technologies, A11037). The total number of infected cells in 4 fields per well with each field containing at least 1000 cells was quantified using an Operetta high-content imaging system (PerkinElmer). Plasma was tested using 2-fold serial dilutions from 1:40 to 1:1280 with 4 replicates per dilution. Results are reported as the highest dilution of plasma leading to at least $50 \%$ reduction of SARS-CoV-2 titers. Each assay was controlled with internal addition of an S-specific neutralizing polyclonal antibody. If a 1:40 dilution did not lead to at least $50 \%$ reduction of viral titer, results were reported as less than 1:40 even though some inhibition of virus propagation may have been present. To enable analysis of nAb kinetics over time, a value of 1:20 was used as a surrogate for results less than 1:40, since this value was the next serial 2-fold titer below the lowest positive result.

$T$ cell assays. To measure $\mathrm{T}$ cell responses to SARS-CoV-2, PBMCs were thawed and plated at $2.5 \times 10^{5}$ to $3 \times 10^{5}$ cells per well in duplicate. Stimuli were 9 pools of SARS-CoV-2 peptides (GenScript) corresponding to the $\mathrm{S}$, membrane $(\mathrm{M})$, and NP proteins of SARS-CoV-2 strain Wuhan (GenBank MN908947.3) in pools of 50-56 peptides per pool. Peptides 13 amino acids long and overlapping by 9 amino acids were used at a final concentration of $1 \mu \mathrm{g} / \mathrm{mL}$ for each peptide and $0.2 \%$ DMSO. Negative control was $0.2 \%$ DMSO; positive control was 1.6 $\mu \mathrm{g} / \mathrm{mL}$ phytohemagglutinin (PHA-P; Remel). IFN- $\gamma$ ELISPOT was performed as previously described (35). All subjects appropriately had too-numerous-to-count positive responses to PHA. Results are reported as spot-forming units per $10^{6} \mathrm{PBMCs}$ for the background-subtracted response to SARS-CoV-2 peptide pools. For subjects with phlebotomy at 2 time points, ELISPOT assays were performed with PBMCs from the second time point only.

Statistics. Univariable analysis was performed using a 2-tailed $t$ test for continuous variables and a $\chi^{2}$ test for categorical variables. Variables with $P \leq 0.2$ in univariable analysis were candidates for multivariable models using logistic regression and were retained in the models if they remained significant or substantially modified the effect of another factor. Statistical significance was defined as 2-sided $P$ less than 0.05. The sensitivity and specificity for high nAb titers by the Abbott and the Euroimmun assays were plotted by generating a receiver operating characteristic (ROC) curve. SPSS software (IBM) was used for statistical analysis and to generate ROC curves, the latter with default parameters. Youden's index was calculated for all points of the ROC curves, and the maximum index value was used to select the optimal $z$ score cutoff (4). Longitudinal analysis of nAb levels was performed using linear mixed effects models with the R package lme4 (36).

Study approval. Institutional Review Board approval was obtained from the University of Washington, and all participants gave written informed consent.

\section{Author contributions}

$\mathrm{JB}, \mathrm{CM}, \mathrm{MKD}, \mathrm{DMK}$, and AW wrote the manuscript with input from all coauthors. AW and TBG conceived the study. JB and AW wrote the IRB protocol. JB, DZ, S McGuffin, and AES recruited participants. SS managed the data. VLC, CLM, and DMK processed specimens. CM, AC, MKD, ALG, KRJ, SLF, LJ, CLM, AB, and MHW characterized and performed antibody and ELISPOT immunoassays. MRH, RG, JL, EP, VVL, and S Mazur performed the neutralization assays. JB, CM, SS, MHW, AW, and DMK performed statistical analysis and generated tables and figures. The order of the 2 co-first authors was determined alphabetically.

\section{Acknowledgments}

We are grateful to all study participants. We thank Angela LeClair, Miko Robertson, Mark Drummond, Kirsten Hauge, Isabelle Hwang, Kristina Chaffee, Avery Forrow, and Tanvi Aggarwal for support in recruiting and enrolling subjects. We thank the members of the University of Washington Clinical Immunology and Virology Labs for conducting the serologic testing. The graphical abstract was created with BioRender.com. The project was funded in part by the Frederick National Laboratory for Cancer Research with support from the National Institute of Allergy and Infectious Diseases under contract 75N91019D00024. This work was also supported by the Fred Hutchinson Joel Meyers Endowment (to JB), Fast-Grants (to JB), a new investigator award from the American Society for Transplantation and Cellular Therapy (to JB), NIH contract 75N93019C0063 (to DMK), NIH T32-AI118690 (to DZ), NIH T32-AI007044 (to S. McGuffin), NIH K08-AI119142 (to SLF), and NIH K23-AI140918 (to AES). The content of this publication does not necessarily reflect the views or policies of the US Department of Health and Human Services or of the institutions and companies affiliated with the authors. This project has been funded in whole or in part with federal funds from the National Institute of Allergy and Infectious Diseases, National Institutes of Health, Department of Health and Human Services, under contract HHSN272201800013C. RG, JL, and MRH performed this work as employees of Laulima Government Solutions LLC. EP, an employee of Tunnell Government Services Inc., is a subcontractor to Laulima Government Solutions LLC.

Address correspondence to: Jim Boonyaratanakornkit, Fred Hutchinson Cancer Research Center, 1100 Fairview Avenue N, Mail Stop E5-320, Seattle, Washington 98109, USA. Phone: 206.667.2379; Email: jboonyar@fredhutch.org. Or to: David M. Koelle, Department of Medicine, University of Washington, 750 Republican Street Room E651, Seattle, Washington 98109, USA. Phone: 206.616.1940; Email: dkoelle@medicine.washington.edu.
1. Casadevall A, Pirofski LA. The convalescent sera option for containing COVID-19. JClin Invest. 2020;130(4):1545-1548.

2. Boonyaratanakornkit J, et al. Primary and repeated respiratory viral infections among infants in rural Nepal. JPediatric Infect Dis Soc. 2020;9(1):21-29.

3. Kiyuka PK, et al. Human coronavirus NL63 molecular epidemiology and evolutionary patterns in rural coastal Kenya. J Infect Dis. 2018;217(11):1728-1739.

4. Youden WJ. Index for rating diagnostic tests. 
Cancer. 1950;3(1):32-35.

5. Weiskopf $D$, et al. Phenotype and kinetics of SARS-CoV-2-specific T cells in COVID-19 patients with acute respiratory distress syndrome. Sci Immunol. 2020;5(48):eabd2071.

6. Joyner MJ, et al. Effect of convalescent plasma on mortality among hospitalized patients with COVID-19: initial three-month experience [preprint]. https://doi.org/10.1101/2020.08.12.2016 9359. Posted on medRxiv August 12, 2020.

7. Kreye J, et al. A SARS-CoV-2 neutralizing antibody protects from lung pathology in a COVID-19 hamster model [preprint]. https:// doi.org/10.1101/2020.08.15.252320. Posted on bioRxiv August 16, 2020.

8. Focosi D, et al. Convalescent plasma therapy for COVID-19: state of the art. Clin Microbiol Rev. 2020;33(4):e00072-20.

9. Atyeo C, et al. Distinct early serological signatures track with SARS-CoV-2 survival. Immunity. 2020;53(3):524-532.e4.

10. Li L, et al. Effect of convalescent plasma therapy on time to clinical improvement in patients with severe and life-threatening COVID-19: a randomized clinical trial. JAMA. 2020;324(5):460-470.

11. Addetia A, et al. Neutralizing antibodies correlate with protection from SARS-CoV-2 in humans during a fishery vessel outbreak with a high attack rate. JClin Microbiol. 2020;58(11):e02107-20.

12. Tortorici MA, et al. Ultrapotent human antibodies protect against SARS-CoV-2 challenge via multiple mechanisms. Science. 2020;370(6519):950-957.

13. Wu Y, et al. A noncompeting pair of human neutralizing antibodies block COVID-19 virus binding to its receptor ACE2. Science. 2020;368(6496):1274-1278.

14. Zost SJ, et al. Potently neutralizing and protective human antibodies against SARS-CoV-2. Nature. 2020;584(7821):443-449.

15. Klein SL, et al. Sex, age, and hospitalization drive antibody responses in a COVID-19 convalescent plasma donor population. J Clin Invest. 2020;130(11):6141-6150.

16. Gudbjartsson DF, et al. Humoral immune response to SARS-CoV-2 in Iceland. N Engl J Med. 2020;383(18):1724-1734.

17. Zhao J, et al. Antibody responses to SARS-CoV-2 in patients of novel coronavirus disease 2019. Clin Infect Dis. 2020;71(16):2027-2034.

18. Long QX, et al. Clinical and immunological assessment of asymptomatic SARS-CoV-2 infections. Nat Med. 2020;26(8):1200-1204.

19. Meckiff BJ, et al. Single-cell transcriptomic analysis of SARS-CoV- 2 reactive $\mathrm{CD} 4^{+} \mathrm{T}$ cells [preprint]. https://dx.doi.org/10.2139/ssrn.3641939. Posted on SSRN July 7, 2020.

20. Wang K, et al. Longitudinal dynamics of the neutralizing antibody response to SARS-CoV-2 infection [published online August 3, 2020]. Clin Infect Dis. https://doi.org/10.1093/cid/ciaa1143.

21. Ibarrondo FJ, et al. Rapid decay of anti-SARSCoV-2 antibodies in persons with mild Covid-19. NEngl J Med. 2020;383(11):1085-1087.

22. Wajnberg A, et al. Robust neutralizing antibodies to SARS-CoV-2 infection persist for months. Science. 2020;370(6521):1227-1230.

23. Rodda LB, et al. Functional SARS-CoV-2-specific immune memory persists after mild COVID-19. Cell. 2021;184(1):169-183.e17.

24. Kreer C, et al. Longitudinal isolation of potent near-germline SARS-CoV-2-neutralizing antibodies from COVID-19 patients. Cell. 2020;182(4):843-854.e12.

25. Kaneko N, et al. Loss of Bcl-6-expressing T follicular helper cells and germinal centers in COVID19. Cell. 2020;183(1):143-157.e13.

26. To KK, et al. COVID-19 re-infection by a phylogenetically distinct SARS-coronavirus-2 strain confirmed by whole genome sequencing [published online August 25, 2020]. Clin Infect Dis. https:// doi.org/10.1093/cid/ciaa1275.

27. McAndrews KM, et al. Heterogeneous anti- bodies against SARS-CoV-2 spike receptor binding domain and nucleocapsid with implications on COVID-19 immunity. JCI Insight. 2020;5(18):e142386.

28. Klasse PJ, Moore JP. Antibodies to SARS-CoV-2 and their potential for therapeutic passive immunization. Elife. 2020;9:e57877.

29. Das MK, et al. Rapid screening evaluation of SARS-CoV-2 IgG assays using z-scores to standardize results. Emerg Infect Dis. 2020;26(10):2501-2503.

30. Kroemer M, et al. COVID-19 patients display distinct SARS-CoV-2 specific T-cell responses according to disease severity [published online August 25, 2020]. J Infect. https://doi. org/10.1016/j.jinf.2020.08.036.

31. Grifoni A, et al. Targets of $\mathrm{T}$ cell responses to SARS-CoV-2 coronavirus in humans with COVID-19 disease and unexposed individuals. Cell.2020;181(7):1489-1501.e15.

32. Schwarzkopf S, et al. Cellular immunity in COVID-19 convalescents with PCR-confirmed infection but with undetectable SARS-CoV-2specific IgG [published online ahead of print October 15, 2020]. Emerg Infect Dis. https://doi. org/10.3201/2701.203772.

33. US Food and Drug Administration. Investigational COVID-19 convalescent plasma (FDA2020-D-1137). Center for Biologics Evaluation and Research.https://www.fda.gov/regulatoryinformation/search-fda-guidance-documents/ investigational-covid-19-convalescent-plasma. May 2020.

34. Wrapp D, et al. Cryo-EM structure of the 2019$\mathrm{nCoV}$ spike in the prefusion conformation. Science. 2020;367(6483):1260-1263.

35. Ott M, et al. T-cell responses to HSV-1 in persons who have survived childhood herpes simplex encephalitis. Pediatr Infect Dis J. 2017;36(8):741-744.

36. Bates DM, et al. Fitting linear mixed-effects models using lme4. J Stat Softw. 2015;67(1):1-48. 